\title{
Overview of W7-X ECRH Results
}

H.P. Laqua ${ }^{1}$, J. Baldzuhn ${ }^{1}$, H. Braune ${ }^{1}$, S. Bozhenkov ${ }^{1}$, K.J. Brunner ${ }^{1}$, Ye.O. Kazakov², S.Marsen ${ }^{1}$, D. Moseev ${ }^{1}$, T.Stange ${ }^{1}$, R.C. Wolf ${ }^{1}$, M. Zanini ${ }^{1}$ and Wendelstein7-X Team

${ }^{1}$ Max-Planck-Institute for Plasma Physics, Greifswald, Germany

${ }^{2}$ Laboratory for Plasma Physics, LPP-ERM/KMS, Brussels, Belgium

Abstract In its second operation phase (OP1.2a) W7-X was equipped with full 3d island divertor and an upgraded ECRH-system of 10 gyrotrons with a total port through power of $7 \mathrm{MW}$. The combination of pellet fueling and high density ECRH enabled to explore density above $110^{20} \mathrm{~m}^{-3}$. In particular with the O2-polarisation combined with a multi-pass reflector tile system a density of up to $1.410^{20} \mathrm{~m}^{-3}$ was achieved. At slightly lower densities high core beta values and record values of the fusion tripple product of $0.6610^{20} \mathrm{~m}^{-3} \mathrm{keVs}$ for stellarators were reached. In addition routine plasma start-up and ECRH wall conditioning were performed. The island divertor enables to demonstrate the intrinsic steady state capabilities of W7-X, where stationary discharges of up to 30s were demonstrated being only limited by the heat capacity of the uncooled divertor. With the flexible ECRH launch system current density profile variations were used for MHD stability investigations. Here by fine-tuning of the ECCD profile different MHD activity could be triggered.

\section{Introduction}

The main objective of the optimized stellarator Wendelstein $7-\mathrm{X}$ (W7-X) is the demonstration of steady-state plasma operation at fusion relevant plasma parameters thereby verifying that the stellarator is a viable fusion power plant concept. The W7-X design is the result on an elaborate optimization procedure which different optimization criteria like neoclassical transport losses, high beta stability, low internal currents and a sufficiently good fast particle confinement at high beta values [1]. W7-X is a low shear stellarator, a concept that avoids the iota profile crossing major rational values. They are positioned at the plasma edge, where the $4 / 5,5 / 5$ or $6 / 5$ island structures are selectively used for the island divertor operation [2].

W7-X was heated by ECRH exclusively in the operation phase OP1.2a. The ECRH system consists of 10 gyrotrons, with an output power of 0.6-1.0 MW each at a frequency of $140 \mathrm{GHz}$, a quasi optical transmission line and flexible microwave launchers inside the plasma vessel [3]. The over-all transmission efficiency was estimated to be $\sim 94 \%$. The ECRH system was commissioned for 1800s operation at full power. It already demonstrated all requirements, which are necessary for a highperformance steady state operation at reactor relevant parameters at $\mathrm{W} 7-\mathrm{X}$. The $140 \mathrm{GHz}$ ECRH used the second harmonic resonance at 2.5T with an absorbed power of up to $7 \mathrm{MW}$. There are in total 12 front steering launchers Inside the plasma vessel, which allow a flexible ECRH launch in both toroidal and poloidal direction for EC current drive (ECCD) and off axis power deposition. In addition W7-X is equipped with two remote steering launchers (see Fig.1), a reactor relevant concept without movable elements in the plasma chamber and an extremely high through-put power density of $100 \mathrm{MW} / \mathrm{m}^{2}$. The high reliability is guaranteed by a FPGA based fast control system, which controls the timing and ratio of the body and cathode voltage at the gyrotrons. In particular it is also able to recognize and recover a gyrotron 
mode loss, which allows a reliably gyrotron operation at maximum power.

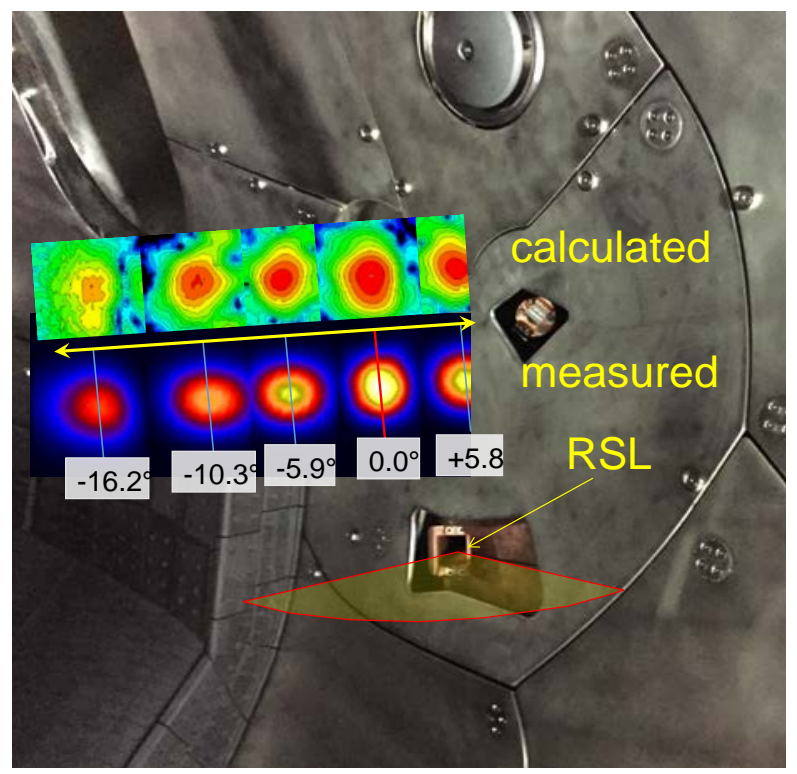

Fig1: Remote steering antenna mouth in the plasma vessel. Comparison of measured and calculated beam pattern for different launch angles.

The W7 -x fue lling system consists of a feedback-controlled gas fueling with valves in each of the 5 W7 -X modules enabling to fuel Hydrogen, Helium and other gases if required. Furthermore W7-X is equipped with a pellet injector, which injected up to 60 hydrogen ice pellets trough both a high -field-side and low field-side injection position.

\section{Plasma start-up and EC-wall conditioning.}

The plasma start -up was exclusively performed by ECRH. The vacuum chamber was prefilled by a gas puff about $100 \mathrm{~ms}$ before the ECRH start. The break-down in the hydrogen or helium gas was achieved within less than $10 \mathrm{~ms}$ with typically 2 gyrotrons beams with $1.7 \mathrm{M} \quad \mathrm{W}$ power in total and focused at the plasma center. Here the ECRHstray radiation was decaying exponentially indicating a well absorbing plasma on the axis, where the ECRH -beams are crossing the second harmonic EC-resonance at 2.5T. This plasma was expanding until it reaches the separatrix. $\mathrm{W}$ ith an additional gas puff the density was then increased to typically 1.5 $10^{19} \mathrm{~m}^{-2}$ within $100 \mathrm{~ms}$ and density feedback system takes over for the regular experimental program. The plasma start -up was successfully achieved from day one with nearly $100 \%$ relia bility for the X2 -heated plasma. In the case of $\mathrm{O} 2$-heated plasmas the central magnetic field had to be slightly increased up to $2.65 \mathrm{~T}$, which necessitated more power for the off -axis start-up. Here the plasma start -up have been achieved within less than 50m s with 3 gyrotrons and $2.5 \mathrm{MW}$ ECRH power . The reliable and efficient operation was guaranteed by a set of ECRH protective diagnostics, which were specially established for the ECRH requirements. In particular the ECRH stray radiation measurement turned out to be a reliable interlock signal to prohibit ECRH operation with insufficient plasma absorption.

Furthermore the position and launch direction of each beam was measured by infrared cameras observing its thermal footprint at the heat shield tiles opposite of the antennas (see Fig.2).

The W7-X plasma chamber was baked-out at $160^{\circ} \mathrm{C}$ for two weeks before the experimental operation. Afterwards glow discharge cleaning with hydrogen and helium was conducted. During the experimental campaign daily helium glow disc harge cleaning was only possible in the morning before the magnetic field was $r$ amped-up. For wall conditioning during the experimental day with a permanent magnetic field of $2.5 \mathrm{~T}$ helium cleaning discharges with ECRH were conducted to remove the otherwise accumulating hydrogen from the plasm awetted surfaces. Several cleaning scenarios were compared. The "pulse train" method turned out as the most effective. Here a sequence of ten $1.5 \mathrm{~s}$ long ECRH -plasmas in Helium for every 30s was used the recovery of density control.

ECCD and long pulse operation. 
W7-X operation does not necessitate toroidal plasma current for operation. Therefore it is not equipped with any tool for inductive plasma current generation. The toroidal plasma current origins only from the bootstrap effect or is non-inductively driven by the ECRH (ECCD). The temporal development of the plasma current is defined by the current diffusion in the plasma (skin time), which is of the order of $1 \mathrm{~s}$, and the $\mathrm{L} / \mathrm{R}$ time of the whole plasma ring, which is of the order of $30 \mathrm{~s}$. Therefore bootstrap current and ECCD studies requires long pulse operation of at least $10 \mathrm{~s}$, but discharge length of up to 30s were achieved. The maximum pulse length was only limited by the heat capacity of the uncooled test divertor tiles. The minimisation of the bootstrap current was one of the main W7-X optimisation criteria. The comparison of the bootstrap evolution in different magnetic configuration verified experimentally the optimisation predictions [4]. Here any ECCD current has to be suppressed, therefore the ECRH launch was precisely to the perpendicular direction at the plasma axis, where the power is absorbed. For the ECCD studies the ECRH beam were steered in oblique direction with an angle of up to $12^{\circ}$ with respect to the perpendicular direction. The polarisation was adjusted adequately to insure pure $\mathrm{X}$-mode injection. The ECCD current was driven in co-and counter direction. A maximum current of 14 kA was achieved after $17 \mathrm{~s}$. This current did not reach its stationary valued yet but was limited by a strong MHD activity with core plasma confinement collapse. We suppose the very localized current density profile deformed the rational transform (iota) profile such that main rational resonance iota $=1$ was crossed and the confinement was destabilized. Detailed theoretical investigations are ongoing. The MHD activity expressed as sawtooth like collapse. The collapse frequency and inversion radius was controlled by localized ECCD. In particular in the so-called high iota configuration, where the iota profile is above but very close to the iota $=1$ value at the core, even small ECCD current generated strong MHD-activity.

\section{High density O2-ECRH operation}

W7-X expressed a rather low density of 0.4 $10^{19} \mathrm{~m}^{-3}$ limit for hydrogen, which is mainly determined by the power balance of the heating power and the radiation at the plasma edge. Even though it is expected that with improved wall conditioning and boronisation less radiation and thus higher densities will be achievable, a separation of core and edge fuelling is beneficial, since both core confinement and divertor performance can be optimized separately.

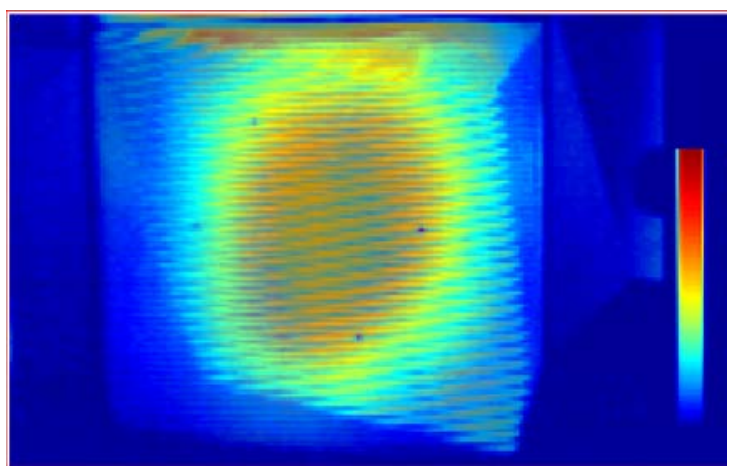

Fig. 2: Infrared footprint of a ECRH beam at the O2-reflector tile with holographic grating at its front.

Therefore W7-X was equipped with an pulsed pellet injector in order to investigate pellet fuelling of ECRH plasmas. With pellet injection the gas fuelling limit could be easily overcome and record densities of up to 1.4 $10^{20} \mathrm{~m}^{-3}$ could be achieved. This density is of course above the cut-off density $\left(1.210^{20} \mathrm{~m}^{-3}\right)$ of the X2 mode for the $140 \mathrm{GHz}$ ECRH. Therefore the second harmonic O-mode $(\mathrm{O} 2)$ with a cut-off density of $2.410^{20} \mathrm{~m}^{-3}$ was be used. Here the single pass absorption was incomplete and no plasma start-up was possible with $\mathrm{O} 2$. Therefore a special $\mathrm{O} 2$ scenario was developed. First the plasma vessel was equipped with tungsten covered molybdenum tiles with holographic gratings 
on its front side as shown in Fig 2. These reflector tiles enabled at least three controlled passes through the plasma core increasing the total absorption to above $90 \%$.

In order to deposit the ECRH power in the hot core and to compensate the plasma beta and Shafranov shift the central magnetic field must be slightly increase up to $2.62 \mathrm{~T}$, which made the plasma start-up even more difficult since now the ECRH power is deposited offaxis at low beta. Therefore as shown in Fig.3 the plasma start-up and the first density ramp was performed by 4 ECRH beam in X2polarisation. During the density ramp-up phase of 2 s the polarisation of these beam had been changed to O2-mode and the density was increased by pellet injection to above the X2 cut-off. At the same time the other O2beam have been switched-on and sustained the high density plasma during the pellet injection over $2 \mathrm{~s}$. In addition to the density measured by Thomson scattering and the laser interferometer, the achievement of the high density was also clearly approved by the vanishing of different ECE-signals due to their cut-off. In Fig. 3 the X2 ECE of the 141 $\mathrm{GHz}$ channel is strongly reduced in the high density phase, while the electron temperature measured by Thomson scattering remains high. It has to be mentioned that this operation is already close to the envisaged future steady state high-performance plasma scenario.

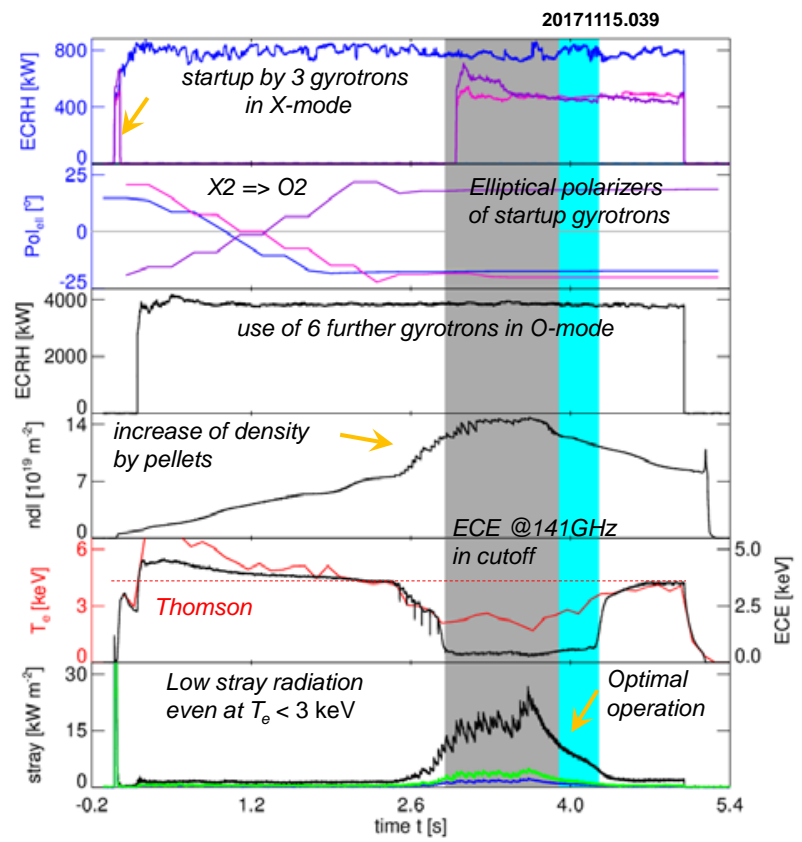

Fig.3: High density plasma scenario with O2ECRH and pellet injection. The second row shows the chance of polarizer position for the 3 "start-up" gyrotron beams, when their polarisation was switched from $\mathrm{X} 2$ to $\mathrm{O} 2$.

\section{High performance discharge.}

At densities close below the X2 cut-off pellet injection was combined also with X2-ECRH (see Fig. 4). Here the discharge scenario was optimized in respect to the highest ion temperature, beta value and tripple product. Therefore just after glow discharge cleaning an X2 heated hydrogen gas fuelled plasma was established at a density of $0.310^{19} \mathrm{~m}^{-3}$. Subsequently, a set of pellets increased the density up to $110^{20} \mathrm{~m}^{-3}$ and the heating power was increased up to $5 \mathrm{MW}$. After the pellet injection in the so-called reheat mode the highest plasma performance was achieved. The electron and ion temperature equilibrated already during the pellet phase and the ion temperature increases up to $3.8 \mathrm{keV}$ with ECRH only. The confinement time increased up to $220 \mathrm{~ms}$ in maximum. The diamagnetic plasma energy reached a record value of 1.1 MJ with a resulting triple product was 0.66 $10^{20} \mathrm{~m}^{-3} \mathrm{keV}$. 


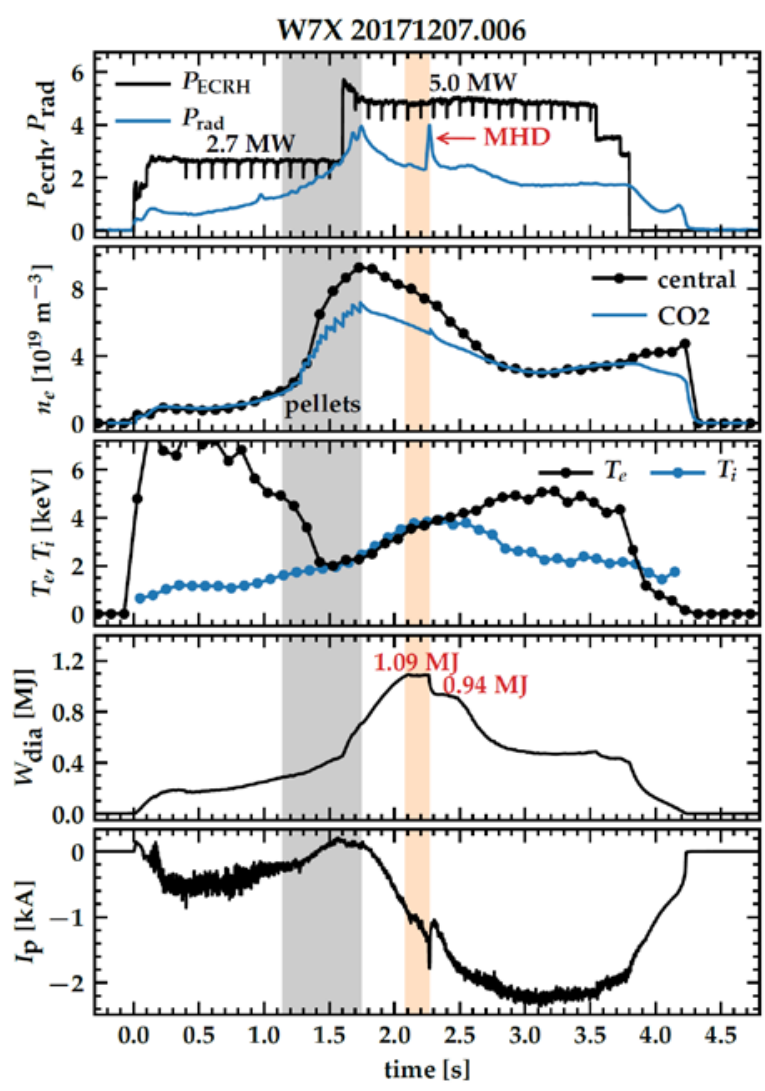

Fig 4: High performance discharge with record beta and triple product. At the top there is the ECRH power and the radiated power. In the next boxes the central Thomson density and the line-averaged density followed by the electron temperature form the Thomson scattering and the ion temperature measured by the $x$-ray spectroscopy are shown. At the fourth row there is the diamagnetic energy and the toroidal plasma current at the bottom.

\section{Collective Thomson Scattering}

The $140 \mathrm{GHz}$ ECRH beams were also used for the collective Thomson scattering (CTS) [5]. Two scattering geometries were available. One in the beam shape section, which uses the movable ECRH front steering launcher, and one in the triangular shape section, which uses the combination of the remote steering launcher for the high power beam and the a quasi-optical remote steering antenna, which was designed by the Stuttgart university, for the receiving beam. The signal transmission towards the detector was performed with an unused ECRH Transmission line in reversed direction. This so-called F1-line is a spare line for further ECRH upgrades. The receiver system, which was built by the Danish technical university (DTU), was placed into the unused F1 gyrotron box. An un-cooled version of the $\mathrm{F} 1$ transmission line was builtup and used to measure the scattering signal. It is remarkable that the $\mu \mathrm{W}$ level CTS signal was partly transmitted with the same multibeam mirrors, that are simultaneously used for the ECRH beams in the MW power level. Nevertheless, no perturbation by the heating beams was detected. The system was successfully commissioned and first ion temperatures were measured. A CTS spectrum is shown in Fig.5.

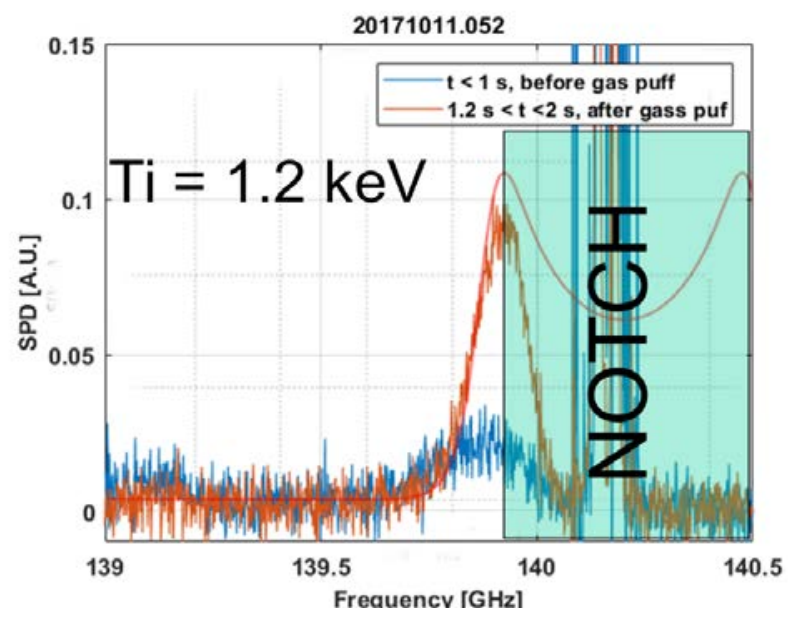

Fig5: CTS spectrum at low density (bleu) and medium density (red) in a Helium plasma.

\section{Summary and conclusion}

The W7-X ECRH system has successfully demonstrated its versatility in the operation phase OP1.2a. It provided the plasma start-up and the wall conditioning routinely. It sustained plasma discharges of up to 30s, which also enabled to demonstrate its ECCD capability. Most remarkable are the results of the combination of ECRH and pellet injection. Here record plasma densities were reached 
with the O2 multi-pass scheme. With X2ECRH slightly below the X2-cutoff density record values of beta, ion temperature and triple product for ECRH plasmas were achieved. Finally the ECRH-beams were also successfully used for the CTS-diagnostic.

\section{References:}

[1] Grieger G. et al Physics optimization of stellarators 1992 Phys. Fluids B4 2081 [2] A. Peacock, et al., Fusion Eng. Des. 84, 1475 (2009).

Progress in the design and development of a test divertor (TDU) for the start of W7-X operation

[3] V. Erckmann et al.(2007) Electron

Cyclotron Heating for W7-X: Physics and Technology, Fusion Science and Technology, 52:2, 291-312, DOI: 10.13182/FST07-A1508 [4].A. Dinklage et al. Magnetic configuration effects on the Wendelstein 7-X stellarator, Nature Physics https://doi.org/10.1038/s41567-018-0141-9

[5] D. Moseev et al. Collective Thomson Scattering Diagnostic at Wendelstein 7-X submitted to review of scientific instruments 